\title{
Cavitation Erosion Prediction on Francis Turbines. Part 2 Model Tests and Flow Analysis
}

\author{
PH. DUPONT, J.-F. CARON, F. AVELLAN \\ EPFL, IMHEF/LMH \\ 33 Av. de Cour, CH-1007 Lausanne, Switzerland \\ P. BOURDON, P. LAVIGNE, M. FARHAT, R. SIMONEAU \\ Hydro-Québec \\ 1800 Boul. Lionel Boulet, Varennes, Québec, Canada J3X ISI \\ J.-M. DOREY, A. ARCHER \\ Electricité de France, DER \\ 6, quai Watier, F-78401 Chatou, France \\ E. LAPERROUSAZ \\ Electricité de France, CNEH \\ Savoie-Technolac, B.P. 26, F-73370 Le Bourget-le-Lac, France \\ M. COUSTON \\ GEC-Alsthom, Neyrpic \\ 75, rue Général-Mangin, B.P. 75, F-38041 Grenoble, France
}

\begin{abstract}
Different measurement techniques have been used to detect cavitation on a Francis turbine model. The results are compared to those obtained on the prototype and presented in the first of this series of articles. The runner model used for that study is build on the basis of a geometrical recovery of one of most eroded blade of the prototype. The results of the different measurements are presented and commented by comparison with prototype measurements. This comparison leads to a proposal of the physics which should be involved in transposition laws for the prediction of prototype erosion from cavitation model tests. The consequences of such scaling laws, as well as their application to the prototype and model results, are part of the third facet of this work.
\end{abstract}

\section{INTRODUCTION}

The problem of cavitation erosion on Francis turbines leads IMHEF, Hydro-Québec, Electricité de France (EDF) and GEC Alsthom-Neyrpic to be involved together in a large and ambitious research program on this topic. Preliminary measurements using various techniques have been performed on the prototype of a $266 \mathrm{MW}$ Francis turbine and are fully described in Part I of this work.

The present paper is concerned with the model tests performed at IMHEF. A runner model is manufactured using the data provided by the geometry recovery of one of the prototype blades [1]. The model measurement campaign, similar to the prototype one, is 
described. As for the prototype measurements, the model runner is equipped with different transducers in order to follow the cavitation development. The results of hydraulic characteristics, vibratory monitoring, pit counting, pressure pulses and electrochemical probes measurements are reported. Additionally, a flow analysis is presented using both Euler and Navier-Stokes calculation codes.

One of the goal of the present study is to define, on the one hand, the best way to perform cavitation tests on model to be as close as possible to the cavitation behavior of the prototype, and one the second hand, the transposition laws to apply to predict cavitation erosion on prototype from the model tests and the way to measure it. The measurements show important differences between model and prototype cavitation development. These differences are commented and analyzed. This leads to propose possible explanations about the physics involved in these scale effects.

In the third part of this series of articles, the similitude and the differences between results of both campaigns are used to give recommendations for the prediction of cavitation erosion from model tests.

\section{GEOMETRY RECOVERY AND DESIGN OF THE MODEL RUNNER}

In order to reproduce the cavitation pattern of the prototype on the model, one of the most eroded blades, namely blade number four, as well as the blade to blade channel corresponding to its suction side have been measured on site. Three techniques were used and compared with each other [1]: the classical template technique, a 3D laser interferometer and a portable 3D digitizer. Because of slight discrepancies between the blade and the blade to blade channel results, it was decided to manufacture the model upon the geometric data of the selected blade. This choice would have lead to bigger outlet openings on the model runner then the measured ones on the prototype. It has been so decided to slightly extend the model blades in onder to keep these openings of the runner identical to the prototype. From this result, a model of the runner with a $300 \mathrm{~mm}$ diameter has been machined using a five axes CNC machine by GEC Alsthom-Neyrpic. To control the possible differences of the flow between model and prototype, flow calculations on the two geometries have been performed.

\section{FLOW CALCULATION}

The analysis of the flow in the model and prototype runner is done using both Euler and Navier-Stokes calculation codes. The geometry recovery of blade four is first used to generate the meshing of the prototype runner. The operating condition followed corresponds to the best efficiency point. The comparison of the results of the different calculation codes used with this prototype geometry is given in left part of Figure 1.

This flow analysis shows that the cavitation development seems to be due to a local bulge of the blade, close to the band and at the third of the blade chord length. This local bulge creates a local underpressure located just upstream of the eroded area of the prototype. Moreover, changing the blade to band fillet size of the tested model runner at suction side has shown to change the cavitation inception. It is so suspected that both 
corner flow along the blade to band fillet together with this local underpressure is responsible for the unexpected erosion observed on the prototype. The influence of the model blade elongation is investigated comparing the pressure distribution calculated for both prototype and model blade geometry. The right part of Figure 1 shows the elongation to slightly increase the pressure level at runner outlet close to the band.
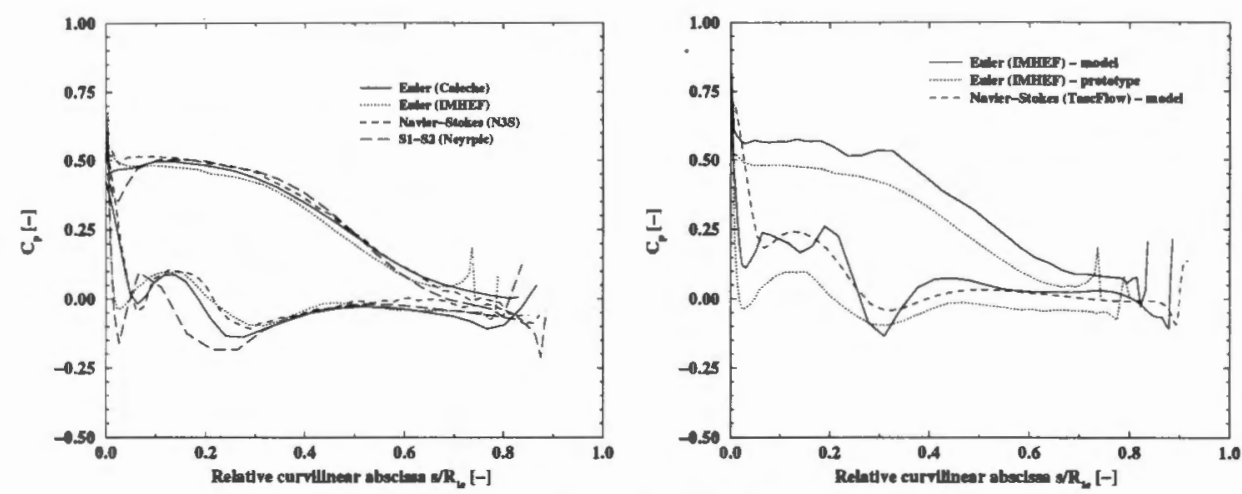

Figure 1: Pressure coefficient distribution along the blade close to the band.

On left: Prototype blade \#4 geometry - Comparison of different codes

On right: Comparison between prototype and model geometry results

\section{MODEL TESTS SET-UP}

The model tests are performed on the high performance research test rig of IMHEF (PF III). This closed loop facility allows to reach flow rates up to 1.5 cubic meters per second, with a maximum net head $\mathrm{H}_{\mathrm{n}}$ of 60 meters. A first series of tests are performed using a set of blades without any instrumentation to compare the hydraulic performances of the model with the prototype measurements.

After this first step, 6 of the 13 blades are replaced with instrumented ones. Two blades (2 \& 8) are equipped each with five miniature static and dynamic pressure transducers, with a useful 15 to $20 \mathrm{kHz}$ upper frequency determined by a preliminary dynamic calibration. These sensors allow to measure the pressure fluctuations acting on the blade due to cavitation. Two other blades (4 \& 10) are instrumented with a total of five DECER electro-chemical cavitation erosion probes, which provide a real time monitoring of the erosion rate. Finally, two blades ( $6 \& 12)$ are equipped with samples of soft material, namely pure copper, for the pit counting experiment. The locations of the first four transducers (pressure, electro-chemical or samples), are identical to those used on the prototype. Moreover, due to the extra length of the model blades, a fifth location is available downstream of the others. These locations correspond to the erosion area found on the prototype, close to the trailing edge of the blades and near to the band to blade fillet. To prevent any disturbance of the flow due to the mounting of the transducers, it was decided to alternate instrumented and non instrumented blades. 


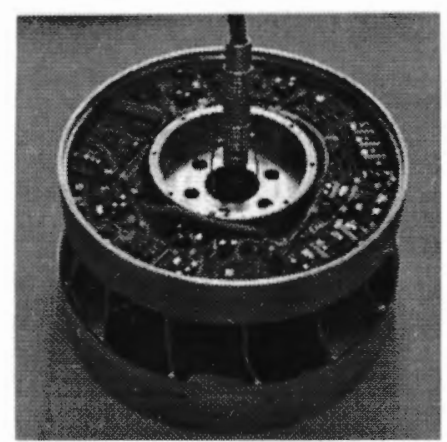

Figure 2: Conditioning electronics

The hub of the runner is especially designed to allow a sealed volume large enough to install the conditioning electronics connected to the onboard transducers (See Figure 2).

This electronics has an amplifier with a remotely variable gain and a multiplexer that allows the scanning of all the transducers, with two simultaneous outputs. Voltage signals are converted to frequency to avoid electromagnetic disturbances. They are brought out from the rotating part through a slip ring collector and finally reverted to voltage signals which are digitized on high speed waveform recorders. In this same sealed volume is placed an accelerometer, fixed to the runner blade ring. This accelerometer replaces for the model tests the one placed on the blade for the prototype tests.

As for the prototype campaign, in addition to the onboard sensors, four accelerometers are used to monitor the cavitation impacts at the lower guide bearing; another one is fixed on the upstream flange of the draft tube cone. The angular position of the last accelerometer is perpendicular to the meridian plane including a dynamic pressure transducer, fixed in the wall of the draft tube cone. The data acquisition systems are essentially the same as those utilized in the prototype program described in part 1 of this series of papers with the addition of a Metrum RSR 51216 channel digital data recorder.

\section{TEST CONDITIONS}

Two operating points are followed during the model tests, corresponding to $78 \%$ and $90 \%$ of the maximum guide vanes opening. To ensure identical runner inlet flow angles on both model and prototype, model guide vanes opening and discharge coefficient $\varphi_{\overline{1} \text { e }}$ are chosen to be equal to the prototype campaign values. Two different model heads, 13 and 51 meters, corresponding respectively to rotational speeds of 800 and 1600 RPM, are investigated to check the influence of the head on the cavitation development. For these two heads, sigma coefficient investigations are done to detect cavitation inception. This inception is determined according to the first pressure peaks detection, corresponding to the first cavity collapses.

Table 1: Operating conditions for the model tests.

\begin{tabular}{ccccc}
\hline TEST & MA01 & MAM1 & MAM2 & MAM3 \\
\hline Guide vanes & 78 & 78 & 78 & 90 \\
opening [\%] & & & & \\
$\sigma_{\overline{1} \mathbf{e}}^{[-]}$ & 0.20 & 0.08 & 0.07 & 0.10 \\
\hline
\end{tabular}

To check the influence of the water nuclei content on the cavitation development, tests were done with and without nuclei injection, using the IMHEF nuclei injection system [2] . If overall cavitation performances are affected by this nuclei content, no influence has been observed on the specific cavitation pattern developing along the blade to band fillet. These specific results are presented in an other paper [3]. To avoid any water quality effects, all measurements are performed after a two hours degassing period. 
The operating conditions used for the pit counting and vibrations tests, given on Table 1 , are similar to the prototype campaign. The sigma values on model are chosen to obtain cavity development in agreement with erosion observed on prototype.

\section{MEASUREMENTS RESULTS}

\subsection{HYDRAULIC CHARACTERISTICS}

\subsubsection{Efficiency tests}

The model results obtained compares very well with those previously obtained with the original model tested by Neyrpic. These hydraulic characteristics, transposed at prototype scale, corresponds quite well to the on site measurements even if two local maximum efficiency are observed on the latest.

\subsubsection{Cavitation tests}

The $\sigma-\eta$ cavitation curves have been performed for the model, and compared to the prototype cavitation behavior. Figure 3 shows the model $\sigma-\eta$ cavitation curve for a $90 \%$ guide vanes opening, and the two sigma values corresponding to the downstream levels achieved during the prototype campaign. The cavitation development on the

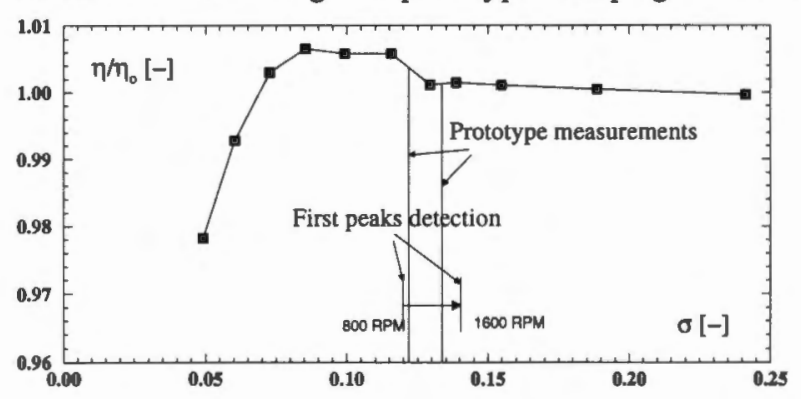

Figure 3: $\quad \sigma-\eta$ cavitation curve at $\alpha=90 \%$. model corresponds to the location of the erosion on the prototype and confirms the local underpressure revealed by the calculation. This seems to indicate that, even if the model is built on the basis of only one blade geometry recovery, the cavitation behavior of the prototype is well reproduced. On the other hand, the comparison of the cavitation index for a given cavity extent, observed on the model and estimated on the prototype from the pitting tests, shows an important deviation. For an equivalent cavitation extent, the corresponding cavitation index is lower on the model than on the prototype. This last point needs a deeper analysis, in the light of a local cavitation coefficient notion presented here latter. It has also been noticed a influence of the fillet size between suction side blade and band on the cavitation inception. Decreasing the size of these fillets, cavitation inception index is notably increased.

\subsection{VIBRATORY MONITORING}

\subsubsection{Dynamic calibration}

To identify an average transmissibility function (ratio of response acceleration to input force autospectra) between the blades and 2 radialy mounted accelerometers at the lower guide bearing, reciprocity instrumented hammer impact techniques [4] are applied between each of these two monitoring points to excite 5 Kistler miniature 
accelerometers mounted on runner blades. Each measurement sequence consists of 32 radialy applied hammer impacts at the guide bearing. A frequency range of $100 \mathrm{kHz}$ with $64 \mathrm{~Hz}$ of resolution is used. The quality of the measurements is excellent as indicated by the coherence function between the input force spectrum and the underwater blade response acceleration spectrum shown in Figure 4 . The transmissibility function is then used in a reciprocity mode to infer forces on the blades from the accelerations measured at the lower guide bearing during the tests.

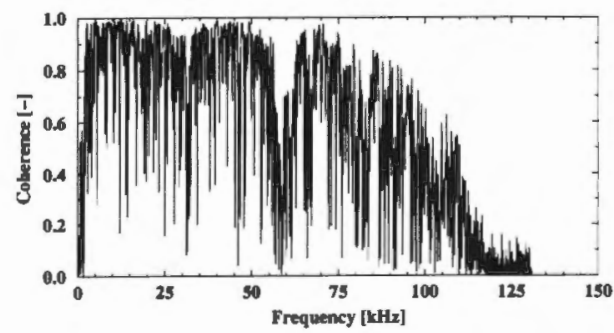

Figure 4: Coherence function between input force and underwater blade response acceleration spectrums.

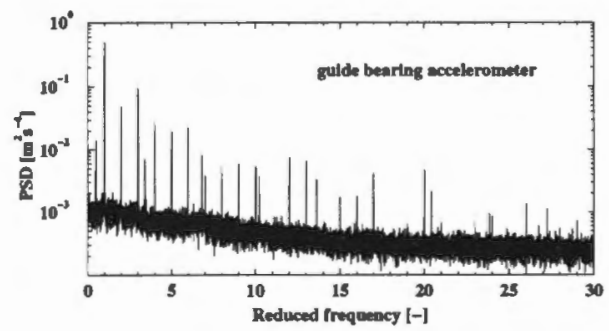

Figure 5: Power spectral density of the 20-35 $\mathbf{k H z}$ accelerations signal envelope at the guide bearing.

A 20 to $35 \mathrm{kHz}$ frequency range is utilized to avoid high frequency interference caused by a 90 degree translating gearbox and to minimize artificial inferred force amplitude caused by the lower resonance frequency of the guide bearing accelerometers in comparison to that of those used on the blades.

\subsubsection{Measurements}

In the test cases, 128 blocs of 4096 points are acquired with Hanning weighting and their autospectra averaged to minimize statistical measurement errors on the random data. So, a $90 \%$ confidence interval ranging better than $+/-1 \mathrm{~dB}$ is achieved at each frequency. Forces acting on the blades are inferred from these autospectra with the transmissibility functions. Forces inferred from the lower guide bearing monitoring accelerometer in the 20 to $35 \mathrm{kHz}$ frequency range are listed in the following table.

Table 2: Inferred forces

\begin{tabular}{cccccc}
\hline TEST & MA01 & MAM1 & MAM2 & MAM2 & MAM3 \\
\hline $\begin{array}{c}\text { Inferred forces } \\
\left(\mathrm{N}^{2}\right)\end{array}$ & 533.6 & 2130.7 & 2101.3 & 1843.8 & 5222.9 \\
\hline
\end{tabular}

High frequency acceleration amplitude modulation studies are also performed on data recorded digitally on the Metrum data recorder. The modulation of the $20-35 \mathrm{kHz}$ acceleration at the different monitoring points is studied by filtering the data with a band pass elliptical filter prior to the envelope detection.

Power spectral densities with Hanning weighting are evaluated with a frequency resolution of $0.0625 \mathrm{~Hz}$. The frequency axis is normalized to orders of rotation. The high frequency vibration envelopes are seen to be mainly modulated by two sets of frequencies centered around the guide vane passing frequency $20 \mathrm{X}$ and the blade passing frequency $13 \mathrm{X}$. The power at these frequencies $+/-1$ and 2 times the rotation frequency are extracted. The power is integrated over a $0.4 \mathrm{~Hz}$ bandwidth around each of these 
frequencies. An example of these PSD is given in Figure 5 showing the forcing effects of these frequencies on the cavitation dynamics.

\subsection{PIT COUNTING MEASUREMENTS}

Three pitting tests (MAM1-2-3) have been performed at the highest head, to ensure the highest pitting rate. These tests are done using $\mathrm{Al} 59$ samples mounted on two different blades. The pitting results are shown on Figure 6 for MAM3 test and blade \#6. The exact flush mounting of the samples is rather difficult due to the blades size. A misalignment has induced side effects corresponding to highest local pitting related to the leading edge of samples, as shown on Figure 6.

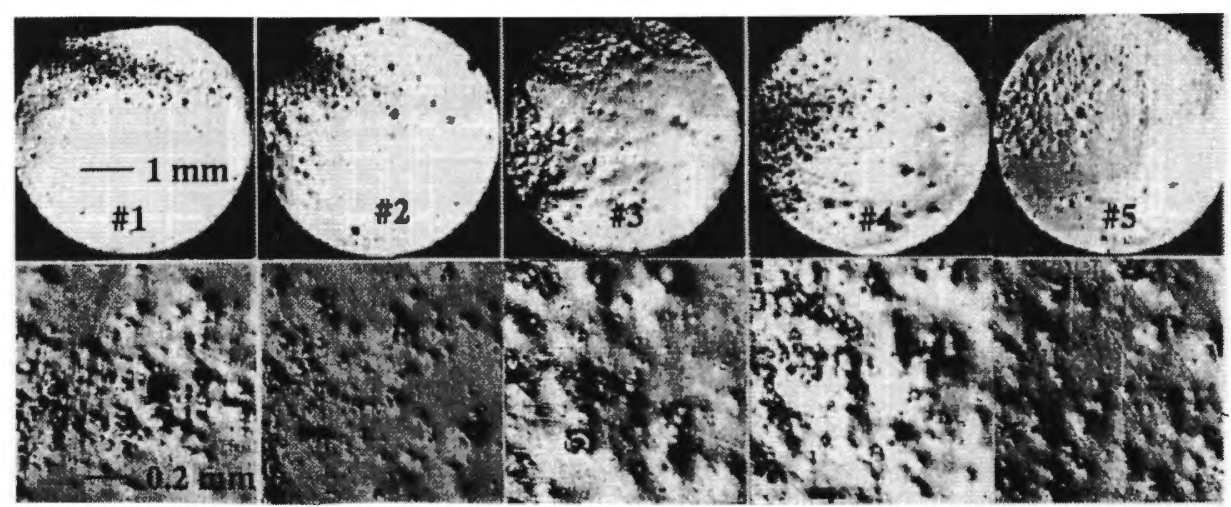

Figure 6: $\quad$ Pitting results on blade \#6 for test MAM3 (Flow direction from left to right)

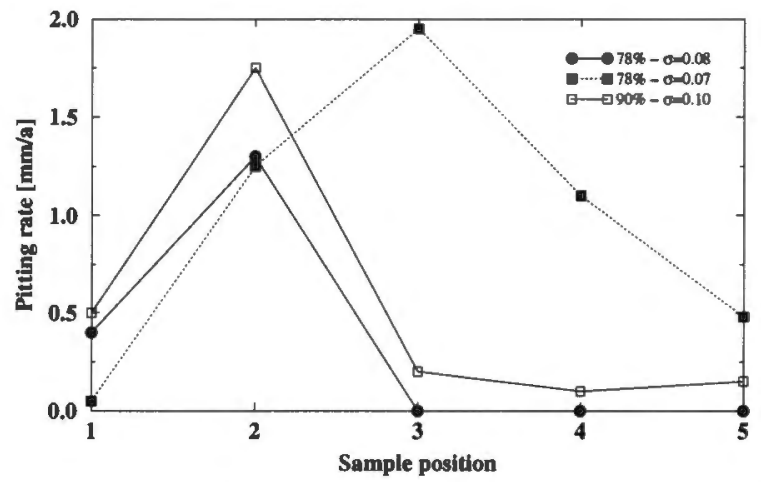

Figure 7: Volume pitting rate for MAM1-2-3 tests using Al 59 samples.

In spite of that, the mean radius of the biggest pits is $0.2 \mathrm{~mm}$, which corresponds to about the tenth of the pits obtained on prototype. This is coherent with the geometric scale ratio equal to 14.67. The volume pitting rates are compared for the three tests on Figure 7. As for the prototype measurements, the maximum pitting is moving closer to the trailing edge of the blade when decreasing the sigma value. As well, a equivalent pitting rate is obtained at the same location and for a highest sigma value for a guide vane opening of $90 \%$ compared with $78 \%$.

\subsection{PRESSURE PULSES MEASUREMENT}

Pressure fluctuations due to cavitation development are measured on two blades on five locations, indicated by dots on Figure 8 . These unsteady pressure transducers are able to detect high frequency pressure variations as shock waves due to cavity collapses. This 
measurement technique is shown to be very efficient to detect the beginning of cavitation. On Figure 8 the incipient cavitation is detect at an opening of $90 \%$ for a $\sigma$ value of 0.132 . Using visual observations, this limit is set for a $\sigma$ value of 0.120 .
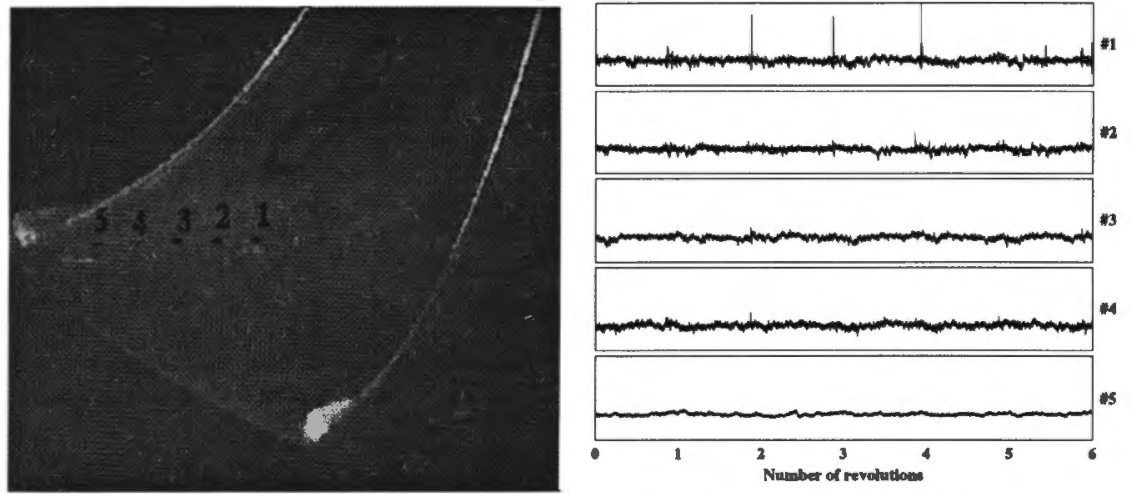

Figure 8: Cavitation pattern visualization and instationnary pressure time signals at five different locations on blade \#8 (Operating conditions: $\alpha=90 \%, \sigma=0.132, \mathrm{H}_{\mathrm{n}}=50 \mathrm{~m}$ ).
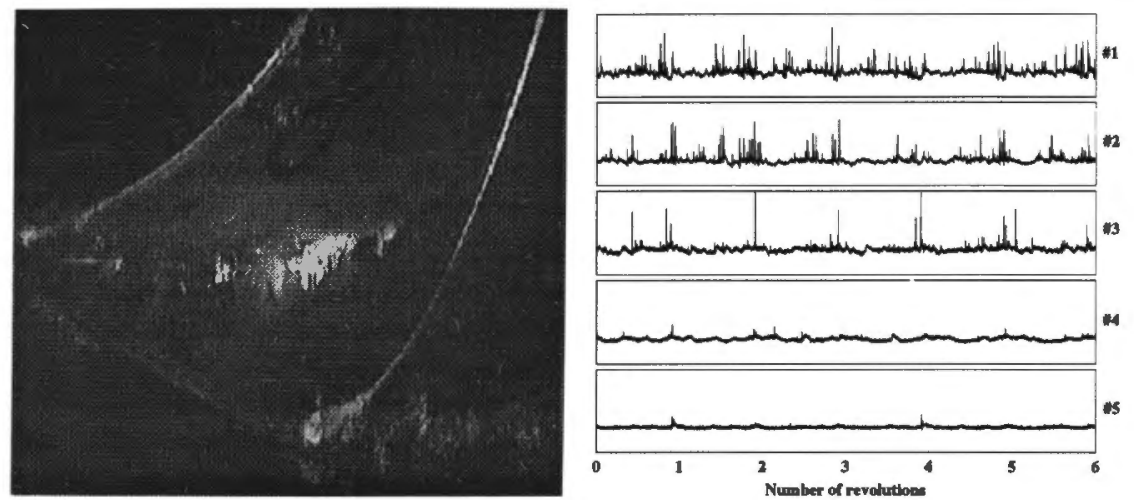

Figure 9: Cavitation pattern visualization and instationnary pressure time signals at five different locations on blade $\# 8$ (Operating conditions: $\alpha=90 \%, \sigma=0.100, H_{n}=50 \mathrm{~m}$ ).

For developed cavitation, pressure measurements gives good information about the modulation of the collapses. On Figure 9 for a $\sigma$ value of 0.100 at $90 \%$ of guide vane opening, a displacement of the collapses closer to the trailing edge is observed. A larger number of small collapses occurs close to pressure transducers \#1 and \#2, while fewer but larger collapses occur close to pressure transducer \#3. This shows the biggest vapor cavities to be convected farer downstream to the local minimum pressure area corresponding to there generation point. It can be observed on Figure 9 the strong modulation of the biggest peaks at each revolution. This will lead for a static observer to vibrations frequency modulation at blade passage frequency as shown by the vibratory monitoring. On the contrary, the guide vane passage frequency is more difficult to detect on pressure fluctuations, may be due to the more intermittent characteristics of this modulation. 


\subsection{ELECTRO-CHEMICAL PROBES MEASUREMENTS}

Because of the small size of the electro-chemical probes and the weakness of the cavity collapses on the model, most of the followed operating points are under the detection threshold of this technique. Only the most erosive situations lead to measurable values corresponding to erosion rates more then three orders of magnitude weaker then those observed on the prototype.

\section{ANALYSIS}

In order to compare the cavitation development on the model and on the prototype, a local cavitation factor $\chi_{\mathrm{E}}$ is used instead of the usual Thoma number $\sigma$. This local cavitation factor is defined as:

$$
\chi_{\mathrm{E}}=\frac{p_{\overline{1 x}}-p_{\mathrm{v}}}{\rho \mathrm{E}}
$$

where $\rho$ is the mass density, $E$ is the energy, $p_{v}$ corresponds to the vapor pressure and $\mathrm{p}_{\overline{1} \mathrm{x}}$ is the local static pressure at the runner outlet.

Using the well known Bernoulli equation between the runner outlet and the draft tube outlet, and taking into account the losses in the draft tube, one can written:

$$
\chi_{\mathrm{E}}=\sigma+\frac{1}{\mathrm{Fr}^{2}} \frac{\mathrm{Z}_{\mathrm{ref}}-\mathrm{Z}_{\overline{1} \mathrm{x}}}{\mathrm{D}_{\overline{1}}}-\frac{\mathrm{C}_{\overline{1} \mathrm{x}}^{2}}{2 \mathrm{E}}+\frac{\mathrm{E}_{\mathrm{rd}}}{\mathrm{E}}
$$

where $\sigma$ is the Thoma number, Fr the Froude number and $E_{r d} / E$ the relative energetic losses in the draft tube. Knowing the flow rate coefficient $\varphi_{\overline{1}}$ at the whirl free operating point $\left(\varphi_{0}\right)$, the energy coefficient at the runner outlet $\Psi_{\overline{1}}$, these relative losses can be expressed as a function of the flow coefficients:

$$
\frac{E_{r d}}{E}=k_{\text {rd }} \frac{\varphi_{\overline{1}}^{2}}{\Psi_{\overline{1}}}+k_{\text {dcin }}\left[\frac{\varphi_{\overline{1}}}{\varphi_{0}}-1\right]^{2} \frac{1}{\Psi_{\overline{1}}}
$$

where $\mathbf{k}_{\mathrm{rd}}$ is the viscous loss coefficient and $\mathbf{k}_{\text {dcin }}$ the residual kinetic energy loss coefficient. Using a model geometrically homologue to the prototype and investigating corresponding flow conditions, the differences in cavitation behavior between model and prototype are due to both Froude and Reynolds effects. The influence of the head on the incipient cavitation is investigated using the pressure transducers. The incipient cavitation index for a net head of 13 and 51 meters, corresponding to a rotational velocity of respectively 800 and 1600 RPM, is indicated on Figure 3. One can noticed that if, at the smallest model head, the first pressure peaks are detected on the model at a $\sigma$ value of 0.120 , lower then the lowest prototype measurement, the incipient cavitation at the highest model head corresponds to a $\sigma$ value of 0.140 , higher then the highest prototype measurement. As the influence of the Froude term is small, this difference is explained by the modification of the relative losses between the two model heads. 


\section{DISCUSSION AND CONCLUSIONS}

The model, build on the basis of one of the most eroded prototype blade (\#4), shows a cavitation development similar to the one suspected on prototype, but occurring for lower $\sigma$ values. This difference is attributed to two main reasons. The first one is the geometry used to build the model. The cavitation behavior at the suction side of a given blade is not only due to its eigen geometry but also to the blade to blade channel geometry. In that meaning, the model is closer to the cavitation developing on the previous blade (\#3), which is one of the less eroded blade on the prototype. The second reason is the scale effects between model and prototype. As shown by the measurements done at 800 and 1600 RPM, Reynolds effects on incipient cavitation are observed. The usage of a local cavitation coefficient leads to much better comparison, but needs to be able to evaluate the different losses on both model and prototype.

The difficulty to compare cavitation erosion on model and prototype is increased by the difficulty to evaluate it on a model. One of the goal of this study is to test different measurement techniques to be used on model to predict erosion on the prototype. The vibratory technique is shown to be well suited to follow developed cavitation, and can be used to quantify erosion rate on model. It is with no doubt the easiest technique to be applied on both model and prototype, because it does not required onboard equipment. It seems on the contrary to be not sensitive enough to detect cavitation inception. The pitting technique on soft material samples gives good information about the localization and the rate of the erosion but it is very sensitive to local geometry defaults. Pressure peaks detection using unsteady pressure transducers is shown to be efficient to detert incipient cavitation as well as the modulation of the collapses. It is on the contrary difficult to be used to quantify the erosion because it is difficult to relate the peaks amplitude to an energy, the location of the collapse being unknown. Finally, electrochemical probes technique, which is able to give on line erosion rate on a prototype, needs to be more sensitive to be used on model.

In closing, the authors would like to thank IMHEF technical staff for their contributions to the success of this project. They are also indebted to Jean-François Combes from EDF/DER, for the Navier-Stokes (N3S) and Euler (Caleche) flow calculations.

\section{REFERENCES}

[1] Dumoulin, Ch., Avellan, F., Henry, P. [et al.], « Geometry recovery of a Francis runner prototype at site ", Proceedings of the 17th IAHR Symposium, section on hydraulic machinery and cavitation, 15-19 September, 1994, Beijing, China

[2] Brand, C., Avellan, F., "The IMHEF system for cavitation nuclei injection ", Proceedings of the 16th IAHR Symposium, section on hydraulic machinery and cavitation, 14-18 September, 1992, Sa0 Paolo, Brasil.

[3] Am, Ch., Dupont, Ph., Avellan, F., «Efficiency alteration of Francis turbine by travelling bubble cavitation: Experimental and theoretical study ", Proceedings of the 18th IAHR Symposium, section on hydraulic machinery and cavitation, 1996, Valencia, Spain.

[4] Bourdon, P., Simoneau, R., Avellan, F., « Erosion Vibratory Fingerprint of Leading Edge Cavitation for a NACA Profile and a Francis Model and Prototype Hydroturbine », Proceedings of the ASME Symposium on Bubble Noise and Cavitation Erosion in Fluid Systems, pp. 51-67, New-Orleans, Dec. 1993 